ARTICLE

DOI: $10.1038 /$ s41467-017-00931-8

\title{
NCoR1 restrains thymic negative selection by repressing Bim expression to spare thymocytes undergoing positive selection
}

Jianrong Wang ${ }^{1}$, Nanhai He², Na Zhang1,3, Dexian Quan¹, Shuo Zhang ${ }^{1}$, Caroline Zhang ${ }^{2}$, Ruth T. Yu (1) ${ }^{2}$, Annette R. Atkins ${ }^{2}$, Ruihong Zhu', Chunhui Yang${ }^{1}$, Ying Cui ${ }^{1}$, Christopher Liddle ${ }^{4}{ }^{4}$, Michael Downes ${ }^{2}$, Hui Xiao ${ }^{1}$, Ye Zheng (1) ${ }^{5}$, Johan Auwerx ${ }^{6}$, Ronald M. Evans ${ }^{2}$ \& Qibin Leng (i) ${ }^{1}$

Thymocytes must pass both positive and negative selections to become mature $T$ cells. Negative selection purges thymocytes whose T-cell receptors (TCR) exhibit high affinity to self-peptide MHC complexes (self pMHC) to avoid autoimmune diseases, while positive selection ensures the survival and maturation of thymocytes whose TCRs display intermediate affinity to self pMHCs for effective immunity, but whether transcriptional regulation helps conserve positively selected thymocytes from being purged by negative selection remains unclear. Here we show that the specific deletion of nuclear receptor co-repressor 1 (NCoR1) in T cells causes excessive negative selection to reduce mature thymocyte numbers. Mechanistically, NCoR1 protects positively selected thymocytes from negative selection by suppressing Bim expression. Our study demonstrates a critical function of NCoR1 in coordinated positive and negative selections in the thymus.

\footnotetext{
${ }^{1}$ CAS Key Laboratory of Molecular Virology \& Immunology, Unit of Immune Regulation, Institut Pasteur of Shanghai, Chinese Academy of Sciences; University of Chinese Academy of Sciences, Shanghai 200031, China. ${ }^{2}$ Gene Expression Laboratory, Salk Institute for Biological Studies, La Jolla, CA 92037, USA. ${ }^{3}$ Obstetrics and Gynecology Hospital, Shanghai Key Laboratory of Female Reproductive Endocrine-related Disease, the Academy of Integrative Medicine, Fudan University, Shanghai 200011, China. ${ }^{4}$ Storr Liver Centre, Westmead Millennium Institute, Sydney Medical School, University of Sydney, Sydney, NSW 2006, Australia. ${ }^{5}$ Immunobiology and Microbial Pathogenesis Laboratory, The Salk Institute for Biological Studies, La Jolla, CA 92037, USA. ${ }^{6}$ Laboratory of Integrative and Systems Physiology, École Polytechnique Fédérale de Lausanne, Station 15, Lausanne CH-1015, Switzerland. Jianrong Wang, Nanhai He and $\mathrm{Na}$ Zhang contributed equally to this work. Ronald M. Evans and Qibin Leng jointly supervised this work. Correspondence and requests for materials should be addressed to R.M.E. (email: evans@salk.edu) or to Q.L. (email: qbleng@sibs.ac.cn)
} 
T hymocytes bearing $\alpha \beta$ T-cell receptors (TCR) must undergo positive and negative selections before becoming mature T cells ${ }^{1-5}$. Positive selection occurs when thymocytes express TCRs with intermediate affinity to self-peptide MHC complexes (self pMHC) that are presented on cortical thymic antigen-presenting cells. Positive selection helps thymocytes to survive and become mature $\mathrm{CD}^{+}$or $\mathrm{CD}^{+}$singlepositive (SP) $\mathrm{T}$ cells $\mathrm{s}^{4,5}$. By contrast, negative selection eliminates $\mathrm{CD} 4^{+} \mathrm{CD} 8^{+}$double-positive (DP) or SP thymocytes expressing TCRs with high affinity to self $\mathrm{pMHCs}^{3}$. Although the strength of the self pMHC-TCR interaction determines the fate of thymocytes undergoing selection, the mechanisms ensuring the survival of thymocytes that are undergoing positive selection remain unclear.

The transcriptional repressor, nuclear receptor co-repressor 1 (NCoR1), has important functions in metabolism, cellular development, and cancer ${ }^{6,7}$. NCoR1 binds to nuclear receptors, including thyroid hormone receptor, retinoic acid receptor and glucocorticoid receptor (GR), and further recruits histone deacetylases (HDAC) to regulate the expression of nuclear receptor target genes ${ }^{6,8}$. Consistent with the function of GR in thymocyte selection ${ }^{9}, 10$, NCoR1 was also shown to have an important function in early T-cell development ${ }^{11}$. Evidently, germline deletion of NCoR1 results in embryonic lethality ${ }^{11}$ and smaller fetal thymi than those of wild-type (WT) controls. Further analysis by fetal thymic organ culture reveals an early blockade of thymocyte development at the $\mathrm{CD}^{-} \mathrm{CD}^{-}$double-negative (DN) $3\left(\mathrm{CD} 25^{+} \mathrm{CD} 44^{-}\right)$stage. However, due to the embryonic lethality induced by NCoR1 deficiency, it is still unclear whether this blockade reflects an intrinsic defect attributable to the loss of NCoR1 in thymocytes, or extrinsic effects from the thymic microenvironment. In addition, how NCoR1 regulates thymocyte development at later stages, particularly during positive and negative selection, remains unknown.

To better define the function of NCoR1 in thymocyte development, we generate T-cell-specific NCoR1-knockout mice. We find that NCoR1 deficiency impacts positive selection by inducing apoptosis in activated thymocytes, resulting in reduced mature $\mathrm{T}$ cells in the thymus and periphery of NCoR1-deficient mice. In addition, NCoR1 deficiency upregulates the expression of Bim, a key mediator of negative selection ${ }^{12}$. Bim-knockout rescues the thymocyte developmental defect in NCoR1-deficient mice, suggesting that Bim may be involved in this positive selection defect. Mechanistically, NCoR1 represses Bim expression by binding to Bim promoter and promoting histone deacetylation. Our data reveal a transcriptional mechanism that protects thymocytes with ongoing positive selection from negative selection, and further suggest that NCoR1 is essential for efficient thymocyte development and optimal peripheral T-cell homeostasis.

\section{Results}

NCoR1 is essential for the late-stage thymocyte development. To better understand how NCoR1 regulates thymocyte development, we first analyzed Ncor1 mRNA expression in different subsets of thymocytes (as well as other tissues). The expression levels of Ncor1 in the thymus were comparable to those in the liver and muscle (Supplementary Fig. 1a). Among the thymocyte subsets, $\mathrm{CD} 4^{+} \mathrm{CD}^{+} \mathrm{DP}$ cells expressed a relatively higher level of Ncorl than $\mathrm{CD}^{-} \mathrm{CD} 8^{-} \mathrm{DN}, \mathrm{CD}^{+}$or $\mathrm{CD}^{+}$SP thymocytes (Supplementary Fig. 1b), indicating that NCoR1 may also play an important role in late-stage thymocyte development, similar to its function in early thymocyte development ${ }^{11}$.

To investigate the cell-type-specific function of NCoR1 in thymocyte development, we generated thymocyte-specific knockout mice by crossing Ncor flox/flox $^{\text {mice }}{ }^{13}, 14$ with
Lck-Cre-recombinase (Cre) or CD4-Cre transgenic mice (to generate $\mathrm{cKO}$ or $4 \mathrm{KO}$ mice, respectively). Ncori flox/flox mice that do not express Cre served as WT controls. Both Ncor1 mRNA and protein levels were decreased by $\sim 70 \%$ in whole thymi of NCoR1-deficient mice compared with WT mice (Figs. 1a and 5d). Regarding the subsets of thymocytes, Ncor1 expression in the DN thymocytes of cKO mice was similar to that in WT mice, but its expression was decreased (by $95 \%$ ) in cKO DP thymocytes (Fig. 1b). NCoR1 deficiency did not significantly alter the total thymocyte number in the thymi of cKO mice (Fig. 1c). By contrast, both CD4 SP and CD8 SP cells from cKO mice were decreased by $\sim 50 \%$ not only proportionally but also in total cell numbers compared with WT or Lck-Cre only mice (Fig. 1d, e and Supplementary Fig. 2a, b). A similar reduction in CD4 SP and CD8 SP thymocytes was also observed in 4KO mice (Supplementary Fig. 3). Further analysis of the maturation status of CD4 SP thymocytes revealed similar proportions of mature $\left(\mathrm{CD} 62 \mathrm{~L}^{\mathrm{hi}} \mathrm{CD} 69^{\mathrm{lo}}\right.$ or $\mathrm{CD} 62 \mathrm{~L}^{\mathrm{hi}} \mathrm{CD} 24^{\mathrm{lo}}$ ) and immature $\left(\mathrm{CD} 62 \mathrm{~L}^{\mathrm{lo}} \mathrm{CD} 69^{\mathrm{hi}}\right.$ or $\mathrm{CD} 62 \mathrm{~L}^{\mathrm{lo}} \mathrm{CD} 24^{\mathrm{hi}}$ ) subsets in cKO mice and WT mice (Fig. 1f, g), indicative of relatively normal SP thymocyte maturation. Thus, NCoR1 deficiency leads to a defect in the transition from DP to SP thymocytes.

To identify whether the defect in NCoR1-deficient mice is intrinsic or extrinsic, we generated chimeric mice by reconstituting irradiated $\mathrm{Rag}^{-1-}$ mice with mixed $\mathrm{cKO}\left(\mathrm{CD} 45.2^{+}\right)$and WT $\left(\mathrm{CD} 45.1^{+} \mathrm{CD} 45.2^{+}\right.$or $\left.\mathrm{CD} 45.1^{+}\right)$bone marrow (BM) cells at a ratio of 1:1. Analysis of the thymocytes at 6 weeks post-reconstitution revealed a significantly lower proportion of $\mathrm{CKO}$ compared with WT (Fig. 1h, i). The percentages of both CD4 SP and CD8 SP thymocytes of cKO mice were more drastically decreased compared with those of WT mice (Fig. 1h, i). Thus, NCoR1 is intrinsically required for the developmental transition from DP to SP thymocytes. Collectively, these data strongly suggest that NCoR1 plays a critical role in the late stages of T-cell development.

NCoR1 deficiency leads to a defect in positive selection. To further explore the stage of thymocyte development that was impaired in NCoR1-deficient mice, we stratified the thymocytes into five developmental stages based on the expression of TCR $\beta$ and CD69 as previously reported ${ }^{15,16}$ (Fig. 2a, b). NCoR1deficient mice exhibited slightly but significantly higher numbers of the two early-stage populations, TCR $\beta^{\mathrm{lo}} \mathrm{CD} 69^{\mathrm{lo}}$ cells (population 1) and TCR $\beta^{\text {int }}{ }^{C} D 69^{\text {lo }}$ cells (population 2), compared with WT mice. Since TCR $\beta^{\text {int }} \mathrm{CD} 69^{\text {lo }}$ cells are primarily pre-selection DP cells, NCoR1 deficiency does not seem to affect the development of pre-selection thymocytes. However, NCoR1-deficient mice had significantly fewer $\mathrm{CD} 69^{+}$thymocytes, in which thymic selection is supposed to occur, including both TCR $\beta^{\text {int }}$ (population 3) and TCR $\beta^{\text {hi }}$ (population 4) populations. Consequently, in the absence of NCoR1, the more mature TCR $\beta^{\text {hi }} \mathrm{CD} 69^{\text {lo }}$ cells (population 5, primarily SP cells), were reduced by approximately $60 \%$ (Fig. 2a, b). It has also been reported that when thymocytes contact self pMHCs on cortical epithelial cells, CD5 expression is upregulated, reflecting the affinity of TCR-self pMHC self pMHCTCR complexes and the strength of the TCR signaling ${ }^{17}, 18$. We found that, similar to the overall decreased expression of CD69 (Fig. 2a), CD5 expression also significantly declined in NCoR1deficient thymocytes (Supplementary Fig. 4a). Further analysis of DP thymocytes revealed that NCoR1 deficiency mainly affected thymocytes undergoing positive selection, because the reduction of CD5 expression only occurred in CD69-positive subpopulations but not in CD69-negative subpopulations (Supplementary Fig. 4b). Taken together, these data suggest that NCoR1 deficiency disrupts the selection process of thymocytes upon contact with self- pMHCs. 
a

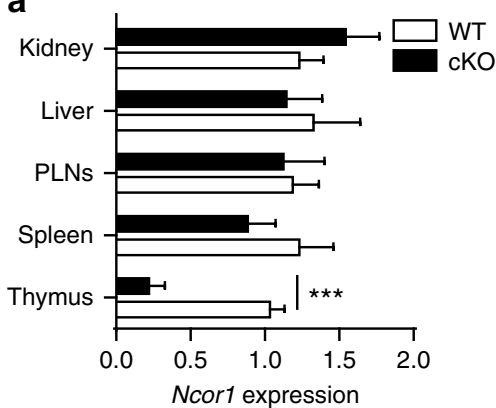

e

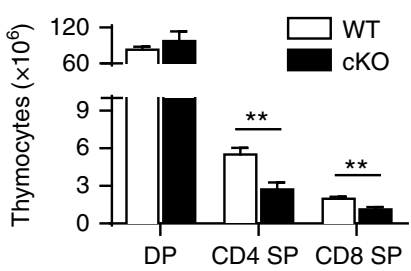

b

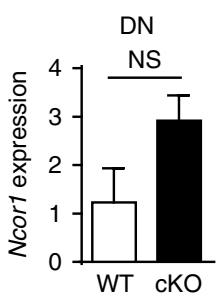

c

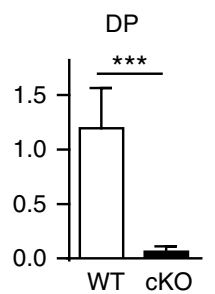

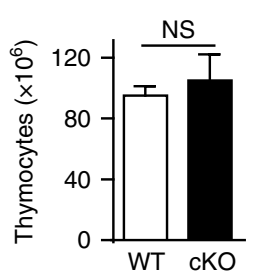

d

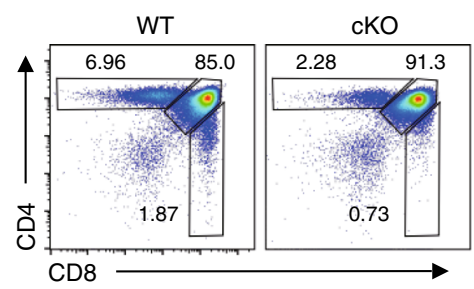

f

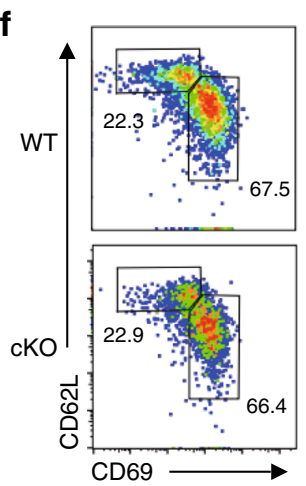

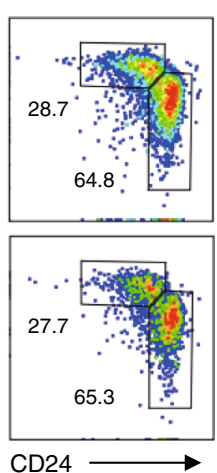

g ○ WT - cKO

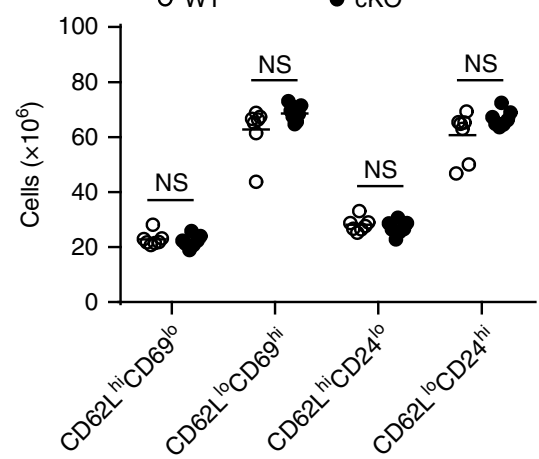

h

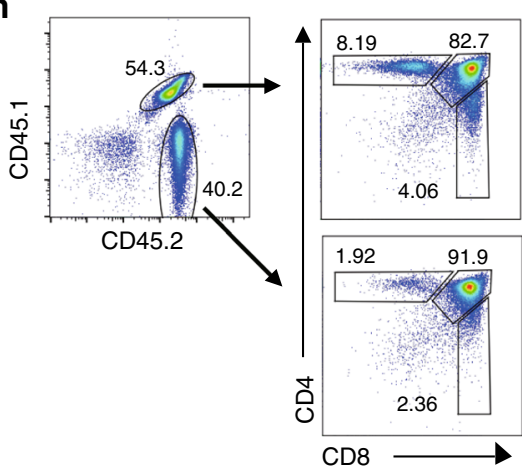

i
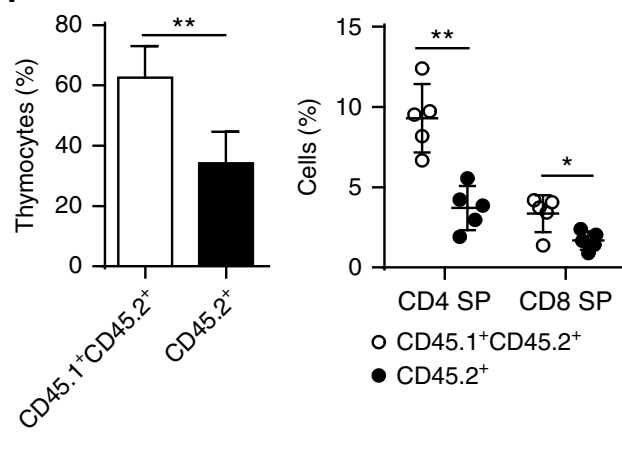

Fig. 1 Late-stage thymocyte development is defective in NCoR1-deficient mice. a, b Quantitative RT-PCR analysis of Ncor1 mRNA levels in indicated tissues $(\mathbf{a}, n=5)$, and sorted $C D 4^{-} C D 8^{-}$double-negative $(D N)$ thymocytes $(n=3)$ and $C D 4^{+} C D 8^{+}$double-positive (DP) ( $\left.n=6\right)(\mathbf{b})$ thymocytes from NCoR1-deficient (cKO) and wild-type (WT) mice. c, d Total cell numbers (c) and surface expression of CD4 and CD8 (d) on thymocytes from cKO ( $n=5$ ) and WT $(n=5)$ mice detected by flow cytometry. e Absolute cell numbers of DP, CD4 ${ }^{+}$or CD8 ${ }^{+}$single-positive (SP) thymocytes from cKO and WT mice ( $n=5$ for each group), as the product of total thymocytes multiplied by the percentage of cells found in that population. $\mathbf{f}$ Expression profiles of CD62L vs. CD69 or CD24 on CKO $(n=3)$ and WT $(n=3)$ CD4 SP thymocytes detected by flow cytometry. $\mathbf{g}$ Cell numbers of each subpopulation gated in $(\mathbf{f})$. h Expression of CD4 and CD8 (right) from mix-bone marrow chimeras generated from CD45.1 $1^{+-}$WT and CD45.2 $2^{+}$cKO (left) donors ( $n=4$ to 5 ). i Quantification of each subpopulation gated in $(\mathbf{h})$. The data are representative of two independent experiments $(\mathbf{a}, \mathbf{b}, \mathbf{h})$ or three independent experiments (c-f). Statistical significance was analyzed using the two-tailed Student's $t$ test $\left({ }^{\star} P<0.05 ;{ }^{\star \star} P<0.01 ;{ }^{\star \star \star} P<0.001\right.$; NS, not significant)

To evaluate whether NCoR1 deficiency affects positive selection, we bred MHC class II-restricted OT-II TCR-transgenic mice with cKO mice. CD4 SP thymocytes of NCoR1-deficient mice were decreased by $\sim 60 \%$ compared with those of WT mice (Fig. 2c). TCR V $\alpha 2^{+}$thymocytes in NCoR1-deficient mice decreased $\sim 60 \%$ compared with those in WT transgenic mice (Fig. 2d). In addition, CD69 expression was decreased by $\sim 70 \%$ in NCoR1-deficient OT-II mice (Supplementary Fig. 4c). A similar reduction was observed in OT-II mice carrying a RAG1 deficiency, in which no other rearranged TCRs are expressed (Fig. 2e, f). Taken together, these findings suggest that NCoR1 is required for positive selection.

The selection of thymic Foxp3 T regulatory cells (tTreg) and invariant natural killer $\mathrm{T}$ (iNKT) cells differs from that of conventional CD4 SP thymocytes because tTregs recognize self- pMHC with a higher affinity than conventional CD4 SP thymocytes and iNKT cells recognize lipid antigens that are presented by CD1d. Therefore, we also analyzed the effects of the NCoR1 deficiency on tTregs and iNKT cells. As shown in Supplementary Fig. 5, both tTregs and iNKT cells in cKO mice were decreased by $\sim 50 \%$ compared with WT mice. The decrease in tTregs mainly resulted from the overall reduction of CD4 SP thymocytes, because the proportion of tTregs in CD4 SP thymocytes of cKO mice was comparable to that of WT mice (Supplementary Fig. 5b). This result indicates that NCoR1 deficiency causes a general defect in thymocyte selection.

NCoR1 deficiency increases thymocyte apoptosis. Attenuated TCR signaling, particularly reduced ERK pathway signaling, leads to impaired positive selection ${ }^{15}, 16,19-21$. To determine whether defective TCR signaling is involved in the decreased positive 
selection in NCoR1-deficient mice, we stimulated thymocytes with anti-TCR $\beta$ and anti-CD28 antibodies, and examined the activation of TCR signaling pathways. We observed similar phosphorylation of PLC $\gamma 1$, Erk1/2 and p38MAPK in NCoR1-deficient and WT thymocytes upon TCR stimulation (Supplementary Fig. 6), suggesting that TCR signaling was not defective in the NCoR1-deficient thymocytes. In addition, the expression level of CD69, an early T-cell activation marker, was comparable between WT and NCoR1-deficient thymocytes in the early stage of in vitro TCR stimulation (Fig. 3a, b), further strengthening the idea that defective positive selection in NCoR1-deficient mice does not result from a defect in TCR signaling.

However, in the late stage of in vitro TCR stimulation, we noticed that CD69 expression on NCoR1-deficient thymocytes was significantly lower than that on WT thymocytes (Fig. 3a, b). As CD69 expression was not affected by the NCoR1 deficiency in the early stage of TCR activation, we therefore sought to examine whether the reduced CD69 expression in the late stage of TCR activation resulted from a loss of activated thymocytes. We found an $\sim 3$-fold increase in apoptotic cell numbers in NCoR1-deficient DP and SP thymocytes compared with WT mice by measuring activated caspase-3 (Fig. $3 \mathrm{c}$ and Supplementary Fig. 7a). Noticeably, apoptotic thymocytes tended to have significantly lower CD69 expression regardless of the NCoR1 deficiency (Supplementary Fig. 7b). Annexin V staining also revealed an increase in apoptosis in NCoR1-deficient thymocytes (Supplementary Fig. 7c, d). Further analyses of ex vivo thymocytes and tissue sections also revealed a significant increase in apoptosis in NCoR1-deficient thymocytes (Fig. 3d-g). These findings suggest that dysregulated apoptosis contributes to the decline in positive selection in NCoR1-deficient mice.

NCoR1 suppresses negative selection. Increased apoptosis indicated that excessive negative selection might contribute to the decline in positive selection in NCoR1-deficient mice. As previously reported, the $\mathrm{CD} 69^{\text {hi }} \mathrm{MHCI}^{-} \mathrm{CD} 4^{\text {dull }} \mathrm{CD} 8^{\text {dull }}$ subset is a distinct fraction of thymocytes that undergoes negative selection $^{12}$. Thus, we examined the effects of NCoR1 deficiency on this particular cell population. Despite the presence of fewer CD69 ${ }^{+}$ thymocytes, NCoR1-deficient mice maintained higher proportions of $\mathrm{CD}_{6} 9^{\text {hi }} \mathrm{MHCI}^{-} \mathrm{CD} 4^{\text {dull }} \mathrm{CD} 8^{\text {dull }}$ cells than WT mice (Fig. $4 \mathrm{a}, \mathrm{b}$ and Supplementary Fig. 2c, d), suggesting that more cells undergo negative selection in NCoR1-deficient mice. In addition, the percentage of activated caspase-3-positive cells was $\sim 2$-fold higher in this subset in NCoR1-deficient mice than in WT mice (Fig. 4c). Thus, NCoR1 is required to suppress negative selection.

To further corroborate the role of NCoR1 in negative selection, we generated chimeric mice by transferring BM cells of OT-II TCR-transgenic WT and cKO mice into irradiated transgenic mice expressing ovalbumin (OVA) under the control of the rat insulin promoter (RIP). Because OVA is expressed in the thymus in this condition, OT-II thymocytes with OVA-specific TCR are forced to undergo negative selection ${ }^{5}$. Our results showed that the thymi of reconstituted mice with NCoR1-deficient OT-II BM cells had an $\sim 4$-fold reduction in the number of CD4 SP and TCR V $\alpha 2^{+}$ thymocytes respectively compared with WT (Fig. 4d, e). These observations indicate that NCoR1 suppresses excessive negative selection.
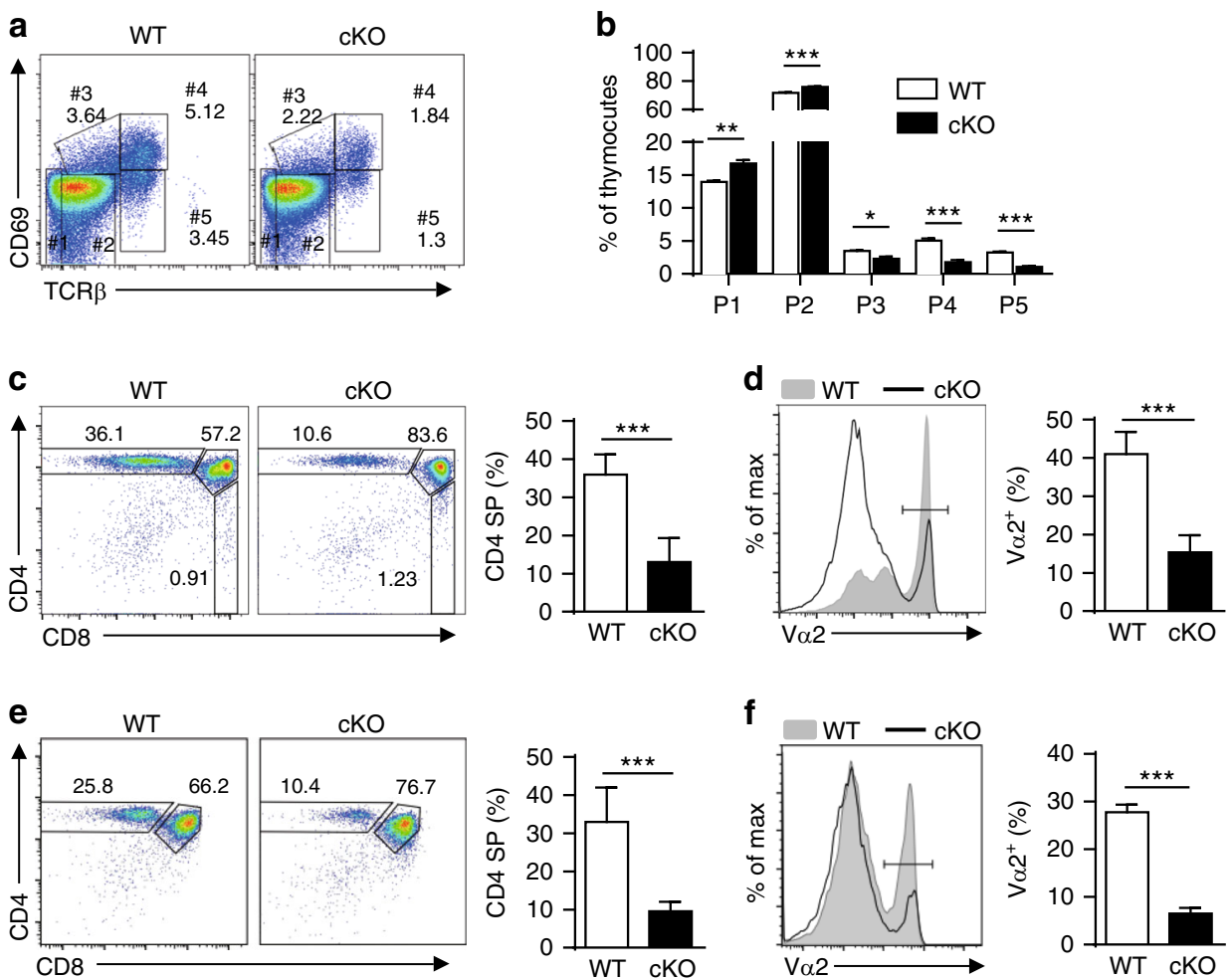

Fig. 2 NCoR1 deficiency leads to a blockade in positive selection. a The surface expression of CD69 and TCR $\beta$ detected by flow cytometry on NCoR1deficient (cKO) and wild-type (WT) thymocytes ( $n=5$ for each group). b Quantification of each subpopulation gated in (a). c, e The expression profiles of CD4 and CD8 (left) on total thymocytes from CKO and WT OT-II TCR-transgenic mice in the Rag $1^{+/+}(\mathbf{c}, n=4)$ or Rag $1^{-/-}(\mathbf{e}, n=3)$ background. The right panel shows the percentages of $\mathrm{CD}^{+}$single-positive (SP) thymocytes gated in the left panel. $\mathbf{d}, \mathbf{f}$ The expression of TCR $V \alpha 2$ detected by flow cytometry on total thymocytes from CKO and WT OT-II TCR-transgenic mice in $\operatorname{Rag}^{+/+}(\mathbf{d}, n=4)$ or $\operatorname{Rag}^{-/-}(\mathbf{f}, n=3)$ background. The right panel shows the quantification of TCR $\vee \alpha 2^{+}$thymocytes gated in the left panel. The data are representative of three independent experiments (a-f). Statistical significance was analyzed using the two-tailed Student's $t$ test $\left({ }^{\star} P<0.05 ;{ }^{\star \star} P<0.01 ;{ }^{\star \star \star} P<0.001\right)$ 
NCoR1 binds to the Bim promoter and represses Bim expression. Given that increased apoptosis is associated with NCoR1 deficiency and that NCoR1 is a transcriptional repressor, we explored whether any proapoptotic genes were de-repressed and hence contributed to the elevated apoptosis of NCoR1-deficient thymocytes. Prior to activation, RNA-Seq analyses revealed 70 genes with significantly changed expression levels between the WT and cKO (28 upregulated and 42 downregulated). Upon activation via TCR ligation, 57 genes were found to be differentially expressed, with 24 upregulated and 33 downregulated genes (Fig. 5a, Supplementary Table 1). Neither CD5 nor CD69 was among the list of differentially expressed genes, likely because the thymocytes expressing these two genes represented only a small fraction of the total thymocytes (Fig. 2a and Supplementary Fig. 4a). Further analysis established that only one proapoptotic gene, $\mathrm{Bim}$, was significantly increased, especially in response to in vitro TCR activation. A similar increase in Bim expression was found in ex vivo-sorted DP thymocytes (Fig. 5b). Consistent with the expression profiling, the expression level of Bim in
NCoR1-deficient thymocytes was upregulated 5-fold 30 min after stimulation (Fig. 5c), and western blotting revealed similar increases in Bim protein (Fig. 5d, e and Supplementary Fig. 8a). Bim expression levels were correlated with the TCR signal strength in both WT and cKO mice (Supplementary Fig. 8a). To further corroborate the results, we also examined Bim expression levels in nonactivated (CD69 negative) pre-selection population $1+2$ and activated population $4+5$ defined in Fig. 2 a. The quantitative RT-PCR analysis revealed that the NCoR1 deficiency only affected Bim expression in the activated population $4+5$ but not in the nonactivated pre-selection population (Supplementary Fig. 8b). Although multiple genes have been reported to promote apoptosis of both thymocytes and T cells, and to participate in negative selection ${ }^{22-25}$, we observed no increases in the expression of Fasl, Trail, Tnf and Bak in NCoR1deficient DP thymocytes before or after in vitro activation (Supplementary Fig. 9). Thus, NCoR1 deficiency specifically upregulates Bim expression in thymocytes, and this elevated expression of Bim, with its known function in negative a

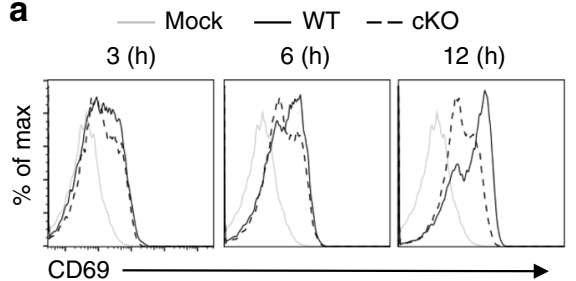

b

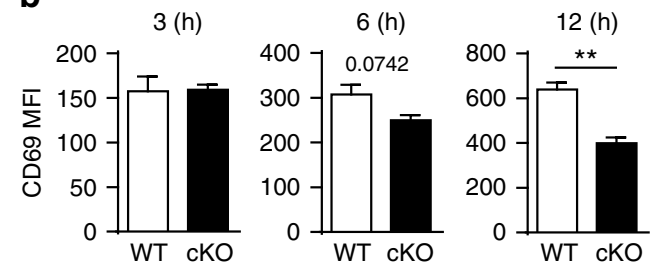

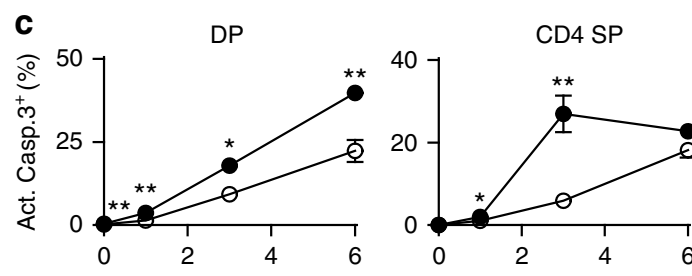

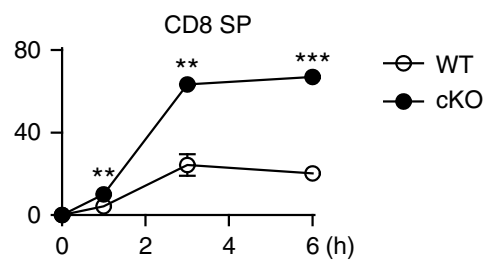

d

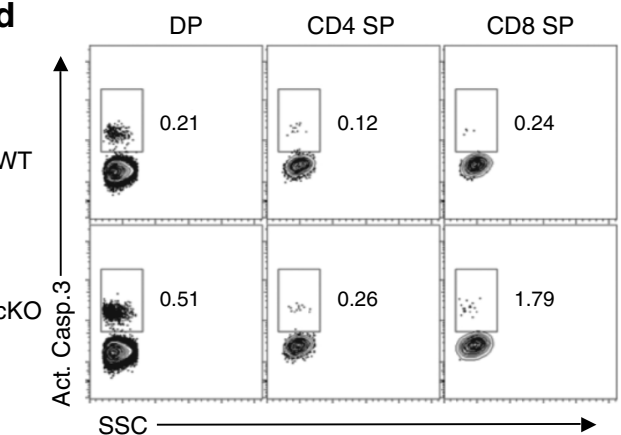

f TUNEL DAPI

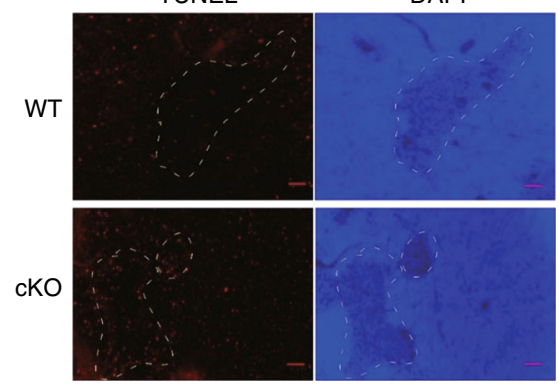

e
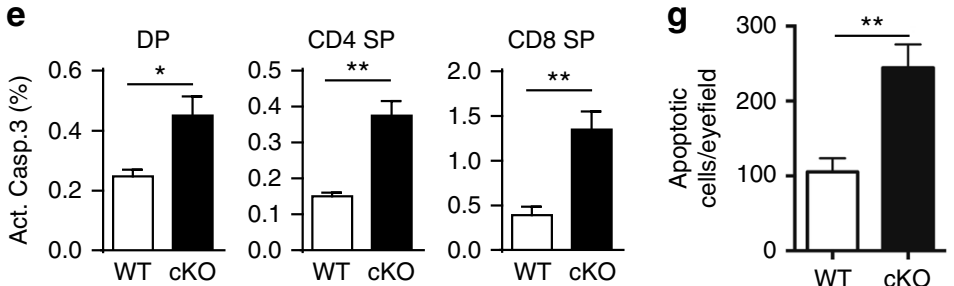

Fig. 3 Deletion of NCoR1 causes elevated thymocyte apoptosis. a Flow cytometry analysis of CD69 expression on sorted in vitro-stimulated CD4 ${ }^{+} \mathrm{CD} 8^{+}$ double-positive (DP) thymocytes from NCoR1-deficient (cKO) and wild-type (WT) mice $(n=3)$. $\mathbf{b}$ The mean fluorescence intensity of CD69 expression in (a). c Intracellular levels of the active caspase-3 (Act.Casp.3) detected by flow cytometry in cKO and WT thymocytes after in vitro TCR stimulation for indicated amounts of time ( $n=3$ for each group). d, e Intracellular levels of the active caspase-3 (Act.Casp.3) detected by flow cytometry in freshly isolated thymocytes ( $n=4$ to 5). $\mathbf{f}$ TUNEL staining of the thymus sections of cKO and WT mice $(n=10)$. The scale bars indicate $100 \mu \mathrm{m}$. $\mathbf{g}$ Quantification of the apoptotic cells in (f). The data are representative of two independent experiments (a-e). Statistical significance was analyzed using the two-tailed Student's $t$ test $\left({ }^{\star} P<0.05 ;{ }^{\star \star} P<0.01 ;{ }^{\star \star \star} P<0.001\right)$ 
selection $^{24-26}$, contributes to the increased apoptosis of activated NCoR1-deficient thymocytes.

Next, we sought to examine whether NCoR1 directly controls the transcriptional expression of Bim. To achieve this goal, we first performed quantitative RT-PCR using chromatin immunoprecipitated with an antibody against NCoR1 in thymocytes (ChIP-PCR). This assay showed that NCoR1 specifically bound to the Bim promoter in WT thymocytes, but not NCoR1-deficient thymocytes (Fig. 5f). In addition, activation of WT thymocytes via TCR ligation led to NCoR1 clearance from the Bim promoter (Fig. 5f), consistent with the increases in Bim mRNA and protein levels in response to TCR activation (Fig. $5 \mathrm{c}-\mathrm{e}$ ). Taken together, our results indicate that NCoR1 binds directly to the Bim promoter to transcriptionally repress its expression.

It is well known that NCoR1 interacts with HDACs such as HDAC3, to promote histone deacetylation. We next analyzed the effects of NCoR1 deficiency on the status of histone acetylation at the Bim promoter by the ChIP assay. NCoR1-deficient thymocytes showed increased acetylation of both histones 3 and 4 compared with WT thymocytes. A similar increase in acetylation specifically on histone $\mathrm{H} 3$ Lys27 (H3K27ac), a marker of active chromatin, was also observed (Fig. 5g, left panel). By contrast, NCoR1 deficiency did not affect the levels of the inactive chromatin markers, mono- and trimethylated $\mathrm{K} 4-\mathrm{H} 3$ histone (Fig. 5g, right panel). These results suggest that NCoR1 is required to maintain the inactive form of chromatin at the Bim promoter by promoting histone deacetylation.

Elevated Bim expression exaggerates negative selection. To assess the presence of a cause-and-effect relationship between
NCoR1 deficiency-induced Bim expression and increased apoptosis during T-cell development, we crossed whole-body Bim KO mice with the NCoR1-deficient mice to generate mice deficient for both the Bim and Ncorl genes (dKO). The proportions of CD4 SP and CD8 SP thymocytes in dKO mice increased 4-10 times compared with mice that were NCoR1-deficient only (Fig. 6a, b). Interestingly, the proportions of SP thymocytes and mature SP thymocytes were comparable between Bim KO and dKO mice (Fig. 6a, c and Supplementary Fig. 10), suggesting that the effects of the NCoR1 deficiency on thymocytes were mainly dependent on Bim. Further analysis of CD69 and CD5 expression in total thymocytes also revealed that the Bim knockout was able to rescue both the decreased CD69 and CD5 expression levels in NCoR1-deficient thymocytes (Fig. 6c, d). Noticeably, compared with WT mice, CD5 expression, CD69 expression, and SP proportions were significantly increased in Bim KO mice, regardless of NCoR1 deficiency (Fig. 6a, c, d). Taken together, these data strongly suggest that Bim deficiency protects against the depletion of both types of thymocytes with ongoing either positive or negative selection.

We also examined the effect of Bim deficiency on the apoptosis of activated NCoR1-deficient thymocytes by performing active caspase-3 staining. Bim deficiency led to significantly reduced apoptosis in activated thymocytes regardless of NCoR1 deficiency. In addition, the proportions of apoptotic cells in $\mathrm{dKO}$ thymocytes did not significantly differ from thymocytes that were deficient for Bim alone (Fig. 6f). Overall, our data suggest that the elevated apoptosis of NCoR1-deficient thymocytes is most likely attributable to dysregulated Bim expression.

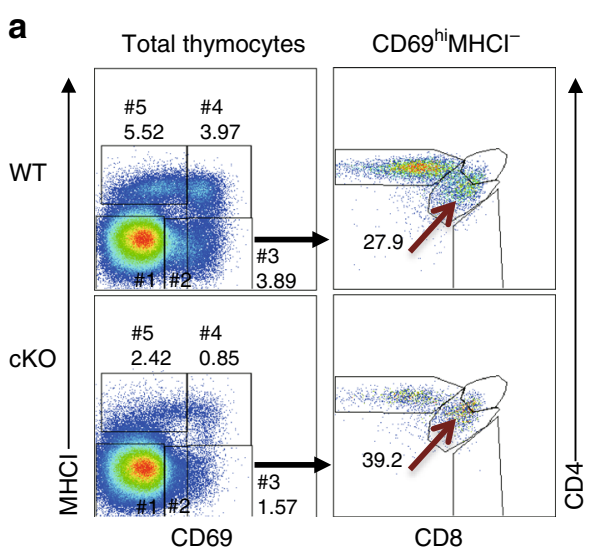

d

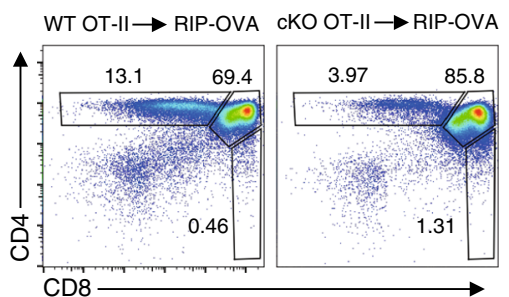

b

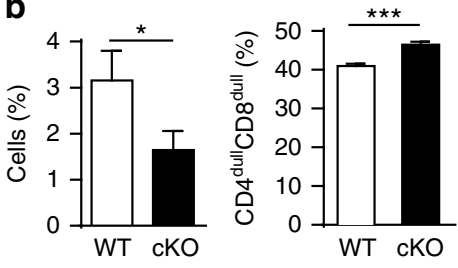

C
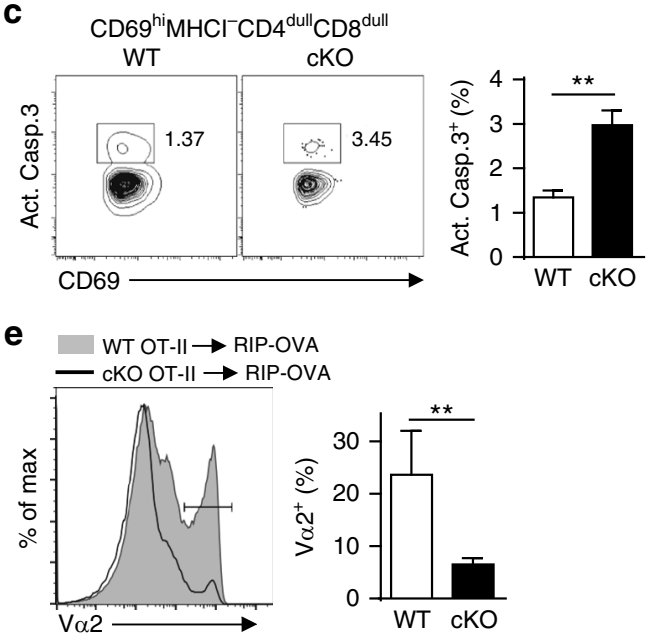

Fig. $4 \mathrm{NCoR} 1$ is required to prevent excessive negative selection. a Flow cytometry analysis of 5 subpopulations (left) by MHCl and CD69 expression on total NCoR1-deficient (cKO) and wild-type (WT) thymocytes ( $n=3$ to 5 ). The right panel shows CD4 and CD8 expression on gated population \#3. b The proportion of population \#3 (left) and CD $4{ }^{\text {dull }} \mathrm{CD} 8^{\text {dull }}$ subsets in population \#3 (right). c Intracellular levels of Act.Casp. 3 detected by flow cytometry in the $\mathrm{CD} 69^{\text {hi }} \mathrm{MHCl}^{-} \mathrm{CD} 4^{\text {dull }} \mathrm{CD} 8^{\text {dull }}$ subsets from $\mathrm{CKO}$ and WT mice ( $n=4$ to 5 for each group). $\mathbf{d}$ Flow cytometry analysis of CD4 and CD8 expression on total thymocytes 6 weeks after transferring bone marrow cells from OT-II TCR-transgenic CKO or WT mice into irradiated rat insulin promoter-ovalbumin (RIP-OVA) transgenic mice ( $n=4$ to 5). e TCR $\vee \alpha 2$ expression of total thymocytes from the mice in (d). The data are representative of three independent experiments $(\mathbf{a}, \mathbf{b})$ or two independent experiments $(\mathbf{c}, \mathbf{d})$. Statistical significance was analyzed using the two-tailed Student's $t$ test $\left({ }^{\star} P<0.05\right.$; ${ }^{\star \star} P<0.01$; $\left.{ }^{\star \star \star} P<0.001\right)$ 
a

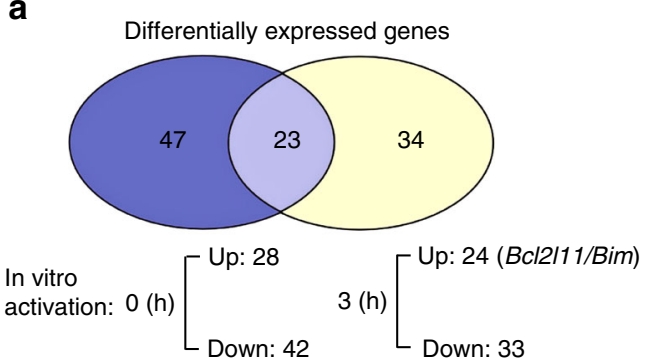

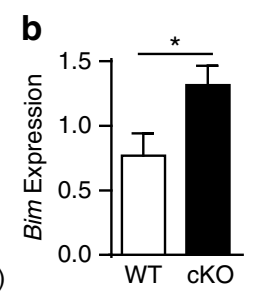

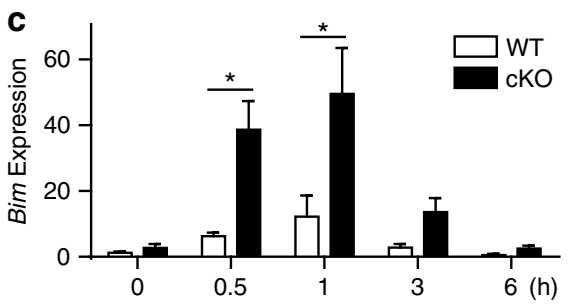

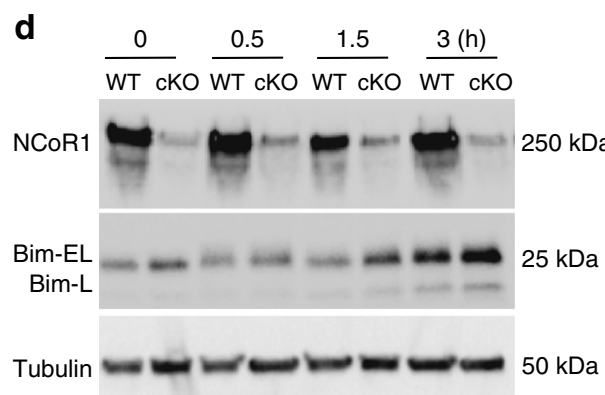
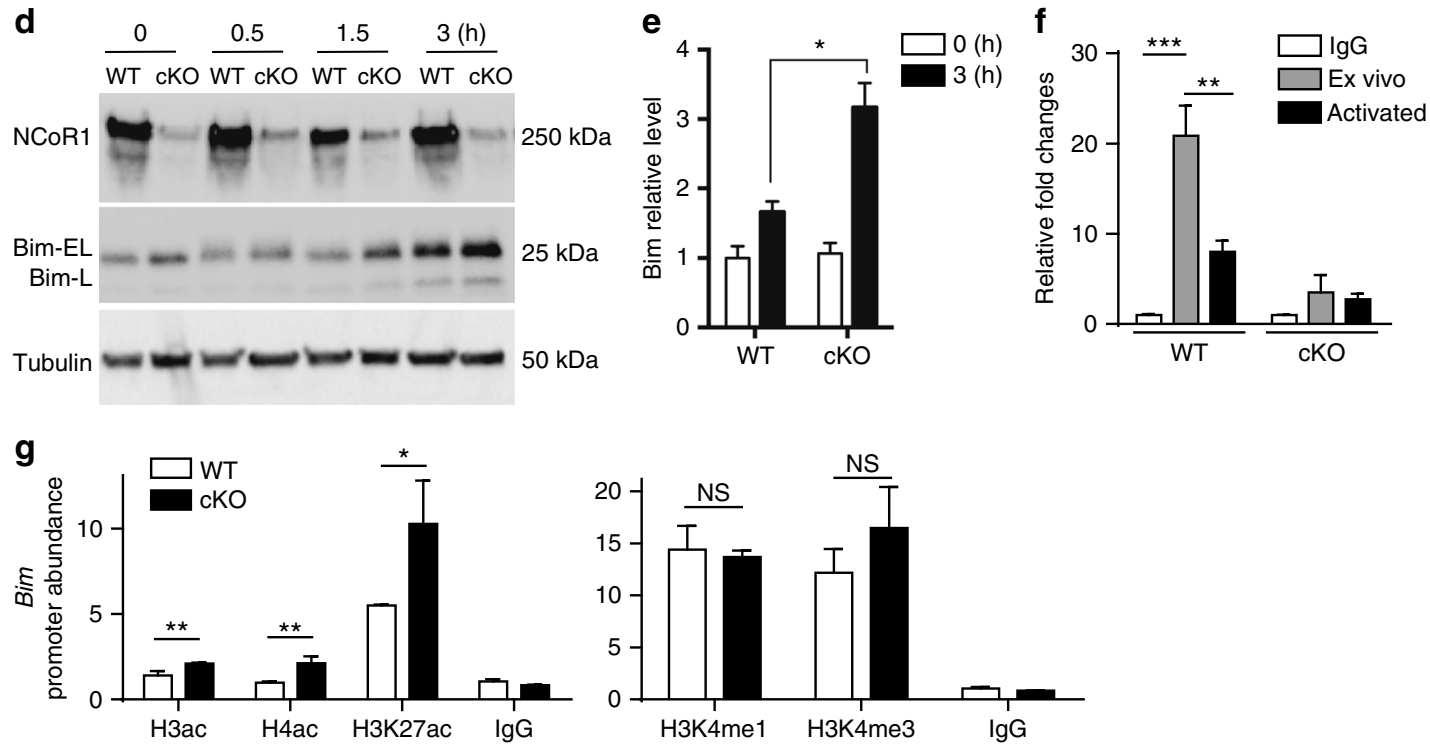

Fig. 5 NCoR1 binds to the Bim promoter and represses Bim expression. a RNA-Seq analysis of differentially expressed genes in NCoR1-deficient (cKO) and wild-type (WT) thymocytes with or without activation in vitro. b, c Quantitative RT-PCR analysis of Bim mRNA expression in freshly sorted cKO ( $n=6$ ) and WT $(n=6) \mathrm{CD}^{+} \mathrm{CD}^{+}$double-positive (DP) thymocytes $(\mathbf{b})$, and in total $\mathrm{CKO}(n=4)$ and WT $(n=4)$ thymocytes (c) after in vitro TCR stimulation for the indicated amounts of time. $\mathbf{d}$ Immunoblot analysis of NCoR1 and Bim expression levels in extracts of cKO and WT thymocytes with or without stimulation for the indicated amounts of time. e Quantification of NCoR1 and Bim expression levels in (d). $\mathbf{f}$ ChIP analysis of the Bim promoter by antiNCoR1 in CKO and WT thymocytes with or without activation in vitro. IgG served as a negative control. $\mathbf{g}$ ChIP analysis of the Bim promoter in cKO and WT thymocytes using the indicted antibodies. The data are representative of two independent experiments $(\mathbf{b}, \mathbf{c}, \mathbf{f}, \mathbf{g})$. Statistical significance was analyzed using the two-tailed Student's $t$ test $\left({ }^{\star} P<0.05 ;{ }^{\star \star} P<0.01 ;{ }^{\star \star \star} P<0.001 ; N S\right.$, not significant)

\section{Discussion}

The fate of immature thymocytes is determined based on the strength of the self pMHC-TCR complexes interaction. Only thymocytes bearing TCRs with intermediate affinity to selfpMHCs undergo positive selection and become mature T cells. By contrast, thymocytes expressing TCRs with high affinity to selfpMHCs will undergo negative selection via apoptosis. The transcriptional regulation that protects thymocytes with ongoing positive selection from negative selection remains unclear. In the present study, we showed that T-cell-specific NCoR1-deficient mice experienced a defect in positive selection, leading to a significant reduction of both mature $\mathrm{CD}^{+}$and $\mathrm{CD}^{+} \mathrm{SP} \mathrm{T}$ cells. This defect was attributable to increased negative selection. Upregulation of Bim expression was associated with increased apoptosis of activated thymocytes. Furthermore, NCoR1 was required for the repression of Bim expression in thymocytes by binding to the Bim promoter. In addition, strong TCR signaling promoted NCoR1 release from the Bim promoter to elicit Bim expression. Bim gene knockout rescued the defective positive selection in NCoR1-deficient mice. In summary, NCoR1 represses Bim expression to protect thymocytes undergoing positive selection from excessive negative selection.
Bim, a proapoptotic molecule, is a key mediator of negative selection during thymocyte development ${ }^{24-26}$. Typically, Bim expression is induced only by a negative selection but not by a positive-selection signal ${ }^{27}$. Although weak positive-selection signals also trigger $\mathrm{Ca}_{2}^{+}$flux ${ }^{27,28}$, only strong TCR activation during negative selection further triggers protein kinase $\mathrm{C}$ activation and the subsequent transcriptional induction of Bim expression $^{27}$. However, the factors that further mediate protein kinase C-dependent Bim transcription in thymocytes were unknown $^{27}$. In this study, we showed that strong TCR signaling released NCoR1 from the Bim promoter, leading to the activation of Bim transcription in WT thymocytes. Furthermore, NCoR1-deficient OT-II mice on RIP-OVA background exhibited a significant increase in negative selection, indicating that NCoR1 indeed inhibited negative selection. Therefore, NCoR1 is likely a protein kinase C-mediated factor that controls Bim expression.

NCoR1 is a large protein with a molecular mass of $\sim 270 \mathrm{kDa}$ and contains a total of three repression domains, two SANT-like domains and three nuclear receptor interaction domains ${ }^{6,}$ 7, 29 . These different domains enable NCoR1 to serve as a platform for interactions with various chromatin-modifying enzymes and 
a

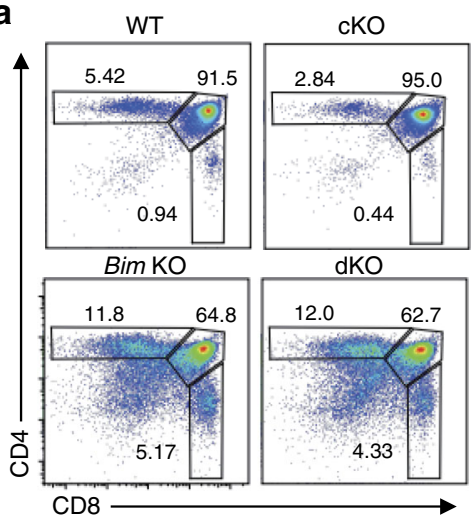

C

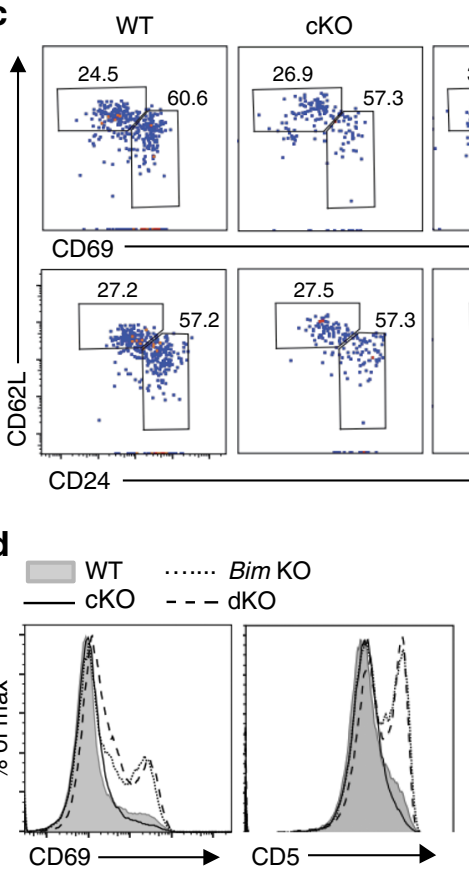

b

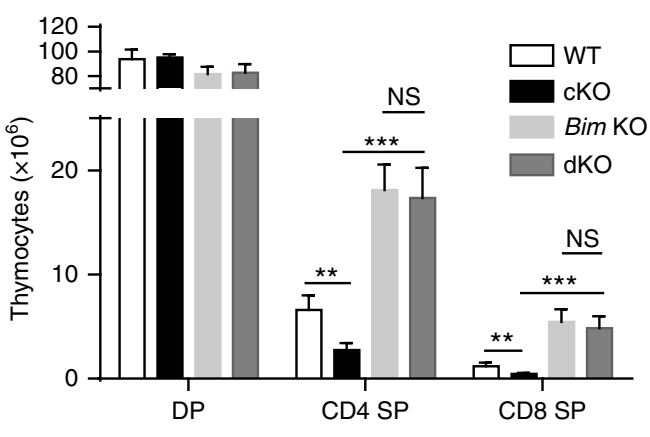

f

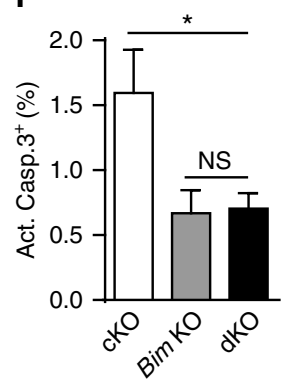

e

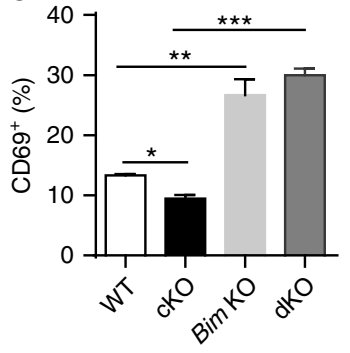

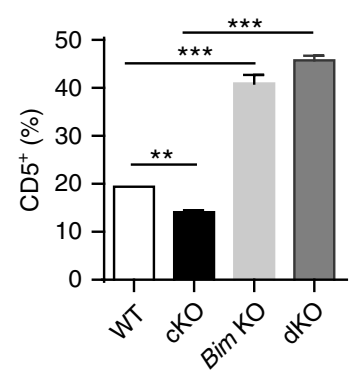

Fig. $6 \mathrm{Bim}$ expression is responsible for the defective thymocyte development in NCoR1-deficient mice. a Flow cytometry analysis of CD4 and CD8 expression on thymocytes from wild-type (WT), NCoR1-deficient (cKO), Bim KO, Ncor1 and Bim double-knockout (dKO) mice. b The number of cells in each subpopulation gated in (a). c Expression of CD62L vs. CD69 or CD24 on CD4 ${ }^{+}$single-positive (SP) thymocytes from WT, cKO, Bim KO, and dKO mice. $\mathbf{d}$ The percentages of CD69- and CD5-positive subpopulations in (c). e Flow cytometry analysis of CD69 and CD5 expression on total thymocytes from WT, cKO, Bim KO, and dKO mice. $\mathbf{f}$ Intracellular levels of the active caspase-3 (Act.Casp.3) in thymocytes activated in vitro for $1 \mathrm{~h}$. The data are representative of two independent experiments $\left(n=2\right.$ or 3 ). Statistical significance was analyzed using the two-tailed Student's $t$ test $\left({ }^{\star} P<0.05\right.$; ${ }^{\star \star} P<0.01 ;{ }^{\star \star \star} P<0.001 ; \mathrm{NS}$, not significant)

transcription factors or nuclear receptors, as well as the formation of dynamic corepressor complexes $6,7,29$. The dynamics and co-occurrence of interacting factors likely contribute to the versatile cell-specific functions of NCoR1 complexes ${ }^{6,29}$. Studies using cell-specific Ncor1 knockout mice show that NCoR1 has distinct functions in adipose tissue, muscle, and macrophages through distinct mechanisms ${ }^{13}, 14,30$, suggesting that NCoR1 has cell- or tissue-specific functions. In the present study, we showed that NCoR1 directly repressed Bim expression and inhibited excessive apoptosis of activated thymocytes, optimizing positive selection in the thymus. In addition, NCoR1-deficient (cKO) mice had only $\sim 50 \%$ of the numbers of peripheral CD4 and CD8 $\mathrm{T}$ cells as WT mice (Supplementary Fig. 11a, b), indicating that NCoR1 may be required for T-cell homeostasis in the periphery. Furthermore, although the proportion of thymic Tregs among CD4 SP thymocytes was comparable to that of WT mice, the proportion of peripheral Tregs among CD4 T cells was increased by $\sim 50 \%$ (Supplementary Fig. 11c, d). This result suggests that NCoR1 may also be involved in the homeostasis of peripheral Tregs. Altogether, NCoR1 most likely has diverse functions in different immune cells.

NCoR1 recruits different HDAC and forms complexes in a context-dependent manner ${ }^{6,7}, 11$. Several studies have revealed that HDAC3 is a major enzyme contributing to the repressive activity of $\mathrm{NCoR} 1^{6,31-33}$. To our surprise, thymocyte development is not defective in HDAC3-floxed CD4-Cre-recombinase (Cre) mice ${ }^{34}$, which is different from the NCoR1-floxed CD4-Cre mice in the present study. Nevertheless, HDAC3-floxed Lck-Cre mice are phenotypically similar to NCoR1-floxed Lck-Cre mice, exhibiting significantly impaired thymocyte development during the $\mathrm{CD} 4^{+} \mathrm{CD}^{+}$DP stage ${ }^{35}$. In addition, $\mathrm{TCR} \beta^{\text {hi }} \mathrm{CD} 69^{\text {hi }}$ thymocytes are also diminished in the absence of HDAC $3^{35}$. Thus, 
similar to NCoR1, HDAC3 may be required for the survival of thymocytes undergoing positive selection. Therefore, HDAC3 may play a role in thymocyte development in both NCoR1dependent and NCoR1-independent manners.

NCoR1 is known to associate with GR and mediate glucocorticoid-induced repression of GR target gene transcription via the assembly of a GR-NCoR1-HDAC3 complex $^{36}$. It is plausible that GR and NCoR1 may play similar roles in T-cell development. An early study revealed that mice carrying a deletion of GR exon 2 displayed normal thymic and peripheral T-cell development ${ }^{37}$. However, other studies revealed that the deletion of GR exon 2 still yielded a truncated product with the ability to regulate gene expression in a ligand-responsive manner ${ }^{38,} 39$. True GR-deleted mice were later generated by two independent groups by conditionally targeting exon $3^{10,40}$. Initially, Baumann et al. claimed that GR-floxed Lck-Cre mice had normal thymocyte development ${ }^{41}$, but Mittelstadt et al. later discovered that GR-floxed Lck-Cre mice had reduced total thymus cellularity due to a partial block in $\mathrm{CD} 4^{-} \mathrm{CD} 8^{-} \mathrm{DN}$ to $\mathrm{DP}$ development ${ }^{10}$. Although the reduction of DP thymocytes was not consistent with our observations in NCoR1-floxed Lck-Cre mice, a similar increase in negative selection associated with a decrease in mature CD4 and CD8 thymocytes was also observed in GR-floxed LckCre mice. In addition, similar Bim upregulation was observed in GR-floxed Lck-Cre mice ${ }^{10}$. Thus, NCoR1 is likely to be involved in the regulatory role of GR during thymocyte selection.

Jepsen and colleagues have reported that NCoR1 expression is essential during the progression of thymocyte development from DN3 stage to DP stage ${ }^{11}$. By contrast, the present study revealed that the T-cell-specific deficiency of NCoR1 did not result in DN3 arrest of thymocyte development. Furthermore, the number of total DP thymocytes in NCoR1 cKO mice was similar to that in WT mice. The ineffectiveness of Ncorl knockout in the DN thymocytes of Lck-Cre mice partially explains this discrepancy. Technically, the previous study is based on the whole-body knockout of NCoR1 and is not able to exclude potential phenotypic contributions of extrinsic effects of NCoR1 deficiency on non-thymocytes. Moreover, because of embryonic lethality, the experiments in that study largely relied on fetal thymic organ cultures rather than live mice. Therefore, the role of NCoR1 in early thymocyte development requires further investigation.

\begin{abstract}
Methods
Mice. All the mice were in C57BL/6 J background. The mouse strain carrying flox alleles of NCoR1 was kindly provided by Dr. Johan Auwerx ${ }^{13}$. These mice were bred with transgenic mice harboring Cre-recombinase driven by the Lck or CD4 promoter to create the following mouse strains: Ncor $1^{\text {floxfflox }}$ (WT), Ncor flox/flox Lck-Cre (cKO) or Ncor 1 flox/flox-CD4-Cre (4KO). Lck-Cre transgenic mice (003802), CD4-Cre mice (022071), Bim ${ }^{-1-}$ mice (004525), Rag1 ${ }^{-1}$ mice (002216), RIP-OVA (Ins2-OVA, 005431) and transgenic mice with the OT-II TCR (003831) were all purchased from the Jackson Laboratory. All mouse strains were bred and maintained under specific pathogen-free (SPF) conditions. Mice, male or female, were used at 6- to 8-week-old, unless otherwise noted. Randomization and blinding strategy was used whenever possible. Mice were killed with carbon dioxide. All mouse experiments followed protocols approved respectively by the Institutional Animal Care and Use Committees in Institut Pasteur of Shanghai and the Salk Institute.
\end{abstract}

\footnotetext{
Flow cytometry. Fluorescence-labeled antibodies for flow cytometry analysis were purchased from eBioscience, Biolegend, and BD Biosciences. Monoclonal antibodies against mouse CD4 (GK15), CD8 $\alpha$ (53-6.7), CD69 (H1.2F3), TCR $\beta$ (H57-597), MHCI (H-2Kb) (AF6-88.5.5.3), CD5 (53-7.3), CD24 (M1/69), CD62L (MEL-14), Foxp3 (FJK-16s) and TCR Va2 (20.1) were from eBioscience. Antibodies against mouse CD45.1 (A20) and CD45.2 (104) were from BD Biosciences or Biolegend. For intracellular staining of Foxp3, cells were fixed and permeabilized with Fixation/Permeabilization Concentrate and Diluent from eBioscience (00-5223-56). Antibodies all above were used at 1:200 dilution. All the experiments were performed using a BD Fortessa or LSR-II flow cytometer (Becton Dickinson). The data were analyzed using FlowJo (TreeStar)
}

Quantitative RT-PCR analysis. Total RNA was extracted from different tissues and cells with TRIzol according to the manufacturer's instructions (Invitrogen), and cDNA was reverse-transcribed from total RNA using the PrimeScript RT Kit (Takara, RR037). Quantitative PCR was performed using the SYBR ${ }^{\circledR}$ Premix Ex Taq $^{\text {tix }}$ II (Takara, RR820) on an ABI 7900HT Fast Real-Time PCR System. The expression of genes was quantitatively normalized to the expression of Actb by the change-in-cycling-threshold $\left(\Delta \Delta \mathrm{C}_{\mathrm{T}}\right)$ method.

BM chimeras. Two million T-cell-depleted BM cells from WT $\left(\mathrm{CD} 45.1^{+} \mathrm{CD} 45.2^{+}\right.$ or $\left.\mathrm{CD} 45.1^{+}\right)$mice and NCoR1-deficient $\left(\mathrm{CD} 45.2^{+}\right)$mice mixed at a ratio of 1:1 were injected into lethally irradiated (600rads, Radsource RS2000 pro) Rag1 ${ }^{-1-}$ mice via tail veins. For RIP-OVA chimeras, BM cells from Ncor $1^{\text {floxfflox }}$-OT-II mice or Ncor $1^{f l o x}$ fflox - Lck-OT-II mice were injected into lethally irradiated (1000rads,

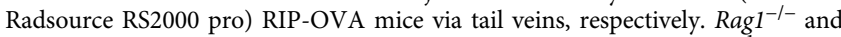
RIP-OVA mice were treated with drinking water containing $2 \mathrm{mg} \mathrm{ml}^{-1}$ neomycin sulfate for 1 week before irradiated. Mice were euthanized and analyzed 6 weeks after the $\mathrm{BM}$ reconstitution.

Apoptosis assays. The apoptosis of ex vivo thymocytes or in vitro-activated thymocytes was detected using the FITC Active Caspase-3 Apoptosis Kit (550480, BD Biosciences) or Annexin V (BMS500FI, eBioscience) according to the manufacturers' guidelines.

TUNEL staining. Thymi were removed and fixed in $4 \%$ paraformaldehyde in phosphate buffer. TUNEL staining was performed using the in situ cell death detection kit according to the manufacturer's protocol of Boehringer Mannheim. After staining, the sections were further counter-stained with haematoxylin.

In vitro thymocyte culture. Total or sorted DP thymocytes were cultured with $5 \mu \mathrm{g} \mathrm{ml}^{-1}$ purified anti-CD28 (37.51, eBioscience) in a 96-well plate that was coated with $10 \mu \mathrm{g} \mathrm{ml}^{-1}$ purified anti-TCR $\beta$ (H57-597, eBioscience) for the indicated times for apoptosis and quantitative RT-PCR analysis.

Immunoblot analysis. A total of 10 to 15 million thymocytes were incubated for $30 \mathrm{~min}$ on ice with anti-CD3 $\left(5 \mu \mathrm{g} \mathrm{ml}^{-1} ; 145-2 \mathrm{C11}\right.$; eBioscience) plus antiCD28 $\left(5 \mu \mathrm{g} \mathrm{ml}^{-1}\right.$; 37.51; eBioscience). After crosslinking with goat antibody to hamster immunoglobulin $\mathrm{G}\left(20 \mu \mathrm{g} \mathrm{ml}^{-1}\right.$; 55397; MP Biomedicals) at various time points, the cells were lysed and subjected to SDS-PAGE. Antibodies for immunoblot were shown in the Supplementary Table 2.

Chromatin immunoprecipitation. The chromatin immunoprecipitation (ChIP) assay was carried out essentially following the manufacturer's protocol (SimpleChIP ${ }^{\circledast}$ Enzymatic Chromatin IP Kit, Agarose Beads, \#9002). Each histone modification ChIP was generated using 20 million cells and the following antibodies: $1 \mu \mathrm{g}$ each of anti-H3ac (06-599, Millipore), anti-H4ac (06-598, Millipore), anti-H3K27ac (ab4729, Abcam), anti-H3K4mel (ab8895, Abcam) and antiH3K4me3 (ab8580, Abcam). Rabbit immunoglobulin G (IgG) was used as a control for nonspecific immunoprecipitation of DNA. For quantitative PCR assays, ChIP DNA was amplified for Bim using forward (5'-CCACCTCTGCCTCTTAAGT AAC- $3^{\prime}$ ) and reverse ( $5^{\prime}$-TCCTCCTTTAGGCTCTCCTTAG-3') primers. The data were analyzed by the following formula: percent $(\%)$ input recovery $=(100 /$ (input fold dilution/bound fold dilution) $) \times 2^{\text {(input CT-bound CT) }}$

RNA isolation and RNA-Seq. Total mouse thymic RNA was isolated using TRIzol (Invitrogen, 15596026) and the RNeasy Mini Kit with on-column DNase digestion (QIAGEN, 74106). RNA purity was assessed using an Agilent 2100 Bioanalyzer. Sequencing libraries were prepared from $100 \mathrm{ng}$ of total RNA using the TruSeq RNA Sample Preparation Kit v2 (Illumina, RS-122) according to the manufacturer's protocol.

Statistical analysis. Statistical analysis of the data (s.d., $t$ tests, one- or two-way ANOVA) was performed using GraphPad Prism version 5.0. Sample sizes were designed with adequate power according to the literature and our previous studies. Randomization and blinding strategy was used whenever possible.

Data availability. The authors declare that the data supporting the findings of this study are available within the article and its supplementary information files, or are available upon reasonable requests to the authors. The RNA-Seq data that support the findings of this study have been deposited at the National Center for Biotechnology Information (NCBI) Sequence Read Archive (SRA) database under the Accession codes\# SRP092624.

Hyperlink:ftp://ftp-trace.ncbi.nlm.nih.gov/sra/review/

SRP092624_20170725_133902_b1659515b9d1a59ebbc790e01084a8f0). 
Received: 29 November 2016 Accepted: 7 August 2017

Published online: 16 October 2017

\section{References}

1. Goldrath, A. W. \& Bevan, M. J. Selecting and maintaining a diverse T-cell repertoire. Nature 402, 255-262 (1999).

2. Palmer, E. \& Naeher, D. Affinity threshold for thymic selection through a T-cell receptor-co-receptor zipper. Nat. Rev. Immunol. 9, 207-213 (2009).

3. Palmer, E. Negative selection--clearing out the bad apples from the T-cell repertoire. Nat. Rev. Immunol. 3, 383-391 (2003).

4. Vrisekoop, N., Monteiro, J. P., Mandl, J. N. \& Germain, R. N. Revisiting thymic positive selection and the mature $\mathrm{T}$ cell repertoire for antigen. Immunity 41, 181-190 (2014).

5. Jameson, S. C., Hogquist, K. A. \& Bevan, M. J. Positive selection of thymocytes. Annu. Rev. Immunol. 13, 93-126 (1995).

6. Mottis, A., Mouchiroud, L. \& Auwerx, J. Emerging roles of the corepressors NCoR1 and SMRT in homeostasis. Genes Dev. 27, 819-835 (2013).

7. Perissi, V., Jepsen, K., Glass, C. K. \& Rosenfeld, M. G. Deconstructing repression: evolving models of co-repressor action. Nat. Rev. Genet. 11, 109-123 (2010).

8. Hua, G., Paulen, L. \& Chambon, P. GR SUMOylation and formation of an SUMO-SMRT/NCoR1-HDAC3 repressing complex is mandatory for GCinduced IR nGRE-mediated transrepression. Proc. Natl Acad. Sci. USA 113, E626-634 (2016).

9. Vacchio, M. S. \& Ashwell, J. D. Thymus-derived glucocorticoids regulate antigen-specific positive selection. J. Exp. Med. 185, 2033-2038 (1997).

10. Mittelstadt, P. R., Monteiro, J. P. \& Ashwell, J. D. Thymocyte responsiveness to endogenous glucocorticoids is required for immunological fitness. J. Clin. Invest. 122, 2384-2394 (2012).

11. Jepsen, K. et al. Combinatorial roles of the nuclear receptor corepressor in transcription and development. Cell 102, 753-763 (2000).

12. Mingueneau, M. et al. The transcriptional landscape of alphabeta T cell differentiation. Nat. Immunol. 14, 619-632 (2013).

13. Yamamoto, H. et al. NCoR1 is a conserved physiological modulator of muscle mass and oxidative function. Cell 147, 827-839 (2011).

14. Li, P. et al. Adipocyte NCoR knockout decreases PPARgamma phosphorylation and enhances PPARgamma activity and insulin sensitivity. Cell 147, 815-826 (2011).

15. Lesourne, R. et al. Themis, a T cell-specific protein important for late thymocyte development. Nat. Immunol. 10, 840-847 (2009).

16. Wang, D. et al. Tespal is involved in late thymocyte development through the regulation of TCR-mediated signaling. Nat. Immunol. 13, 560-568 (2012).

17. Azzam, H. S. et al. CD5 expression is developmentally regulated by T cell receptor (TCR) signals and TCR avidity. J. Exp. Med. 188, 2301-2311 (1998).

18. Yang, Y. et al. The E47 transcription factor negatively regulates CD5 expression during thymocyte development. Proc. Natl Acad. Sci. USA 101, 3898-3902 (2004).

19. Gallo, E. M. et al. Calcineurin sets the bandwidth for discrimination of signals during thymocyte development. Nature 450, 731-735 (2007).

20. Fu, G. et al. Themis controls thymocyte selection through regulation of $\mathrm{T}$ cell antigen receptor-mediated signaling. Nat. Immunol. 10, 848-856 (2009).

21. Johnson, A. L. et al. Themis is a member of a new metazoan gene family and is required for the completion of thymocyte positive selection. Nat. Immunol. 10, 831-839 (2009)

22. Castro, J. E. et al. Fas modulation of apoptosis during negative selection of thymocytes. Immunity 5, 617-627 (1996).

23. Sprent, J. \& Kishimoto, H. The thymus and negative selection. Immunol. Rev. 185, 126-135 (2002).

24. Bouillet, P. et al. BH3-only Bcl-2 family member Bim is required for apoptosis of autoreactive thymocytes. Nature 415, 922-926 (2002).

25. Sohn, S. J., Thompson, J. \& Winoto, A. Apoptosis during negative selection of autoreactive thymocytes. Curr. Opin. Immunol. 19, 510-515 (2007).

26. Ouyang, W., Beckett, O., Ma, Q. \& Li, M. O. Transforming growth factor-beta signaling curbs thymic negative selection promoting regulatory $\mathrm{T}$ cell development. Immunity 32, 642-653 (2010).

27. Cante-Barrett, K., Gallo, E. M., Winslow, M. M. \& Crabtree, G. R. Thymocyte negative selection is mediated by protein kinase $\mathrm{C}$ - and $\mathrm{Ca} 2+$-dependent transcriptional induction of bim [corrected]. J. Immunol. 176, 2299-2306 (2006).

28. Neilson, J. R., Winslow, M. M., Hur, E. M. \& Crabtree, G. R. Calcineurin B1 is essential for positive but not negative selection during thymocyte development. Immunity 20, 255-266 (2004).

29. Baymaz, H. I., Karemaker, I. D. \& Vermeulen, M. Perspective on unraveling the versatility of 'co-repressor' complexes. Biochim. Biophys. Acta 1849, 1051-1056 (2015).

30. Li, P. et al. NCoR repression of LXRs restricts macrophage biosynthesis of insulin-sensitizing omega 3 fatty acids. Cell 155, 200-214 (2013)
31. Gardner, K. E., Allis, C. D. \& Strahl, B. D. Operating on chromatin, a colorful language where context matters. J. Mol. Biol. 409, 36-46 (2011).

32. Sun, Z. et al. Deacetylase-independent function of HDAC3 in transcription and metabolism requires nuclear receptor corepressor. Mol. Cell 52, 769-782 (2013).

33. You, S. H. et al. Nuclear receptor co-repressors are required for the histonedeacetylase activity of HDAC3 in vivo. Nat. Struct. Mol. Biol. 20, 182-187 (2013).

34. Hsu, F. C. et al. Histone deacetylase 3 is required for t cell maturation. J. Immunol. 195, 1578-1590 (2015).

35. Stengel, K. R. et al. Histone deacetylase 3 is required for efficient T cell development. Mol. Cell Biol. 35, 3854-3865 (2015).

36. Zamir, I. et al. A nuclear hormone receptor corepressor mediates transcriptional silencing by receptors with distinct repression domains. $\mathrm{Mol}$. Cell Biol. 16, 5458-5465 (1996).

37. Purton, J. F. et al. Glucocorticoid receptor deficient thymic and peripheral T cells develop normally in adult mice. Eur. J. Immunol. 32, 3546-3555 (2002)

38. Cole, T. J. et al. GRKO mice express an aberrant dexamethasone-binding glucocorticoid receptor, but are profoundly glucocorticoid resistant. Mol. Cell. Endocrinol. 173, 193-202 (2001).

39. Mittelstadt, P. R. \& Ashwell, J. D. Disruption of glucocorticoid receptor exon 2 yields a ligand-responsive C-terminal fragment that regulates gene expression. Mol. Endocrinol. 17, 1534-1542 (2003).

40. Tronche, F. et al. Disruption of the glucocorticoid receptor gene in the nervous system results in reduced anxiety. Nat. Genet. 23, 99-103 (1999).

41. Baumann, S. et al. Glucocorticoids inhibit activation-induced cell death (AICD) via direct DNA-dependent repression of the CD95 ligand gene by a glucocorticoid receptor dimer. Blood 106, 617-625 (2005).

\section{Acknowledgements}

We thank B. Henriquez, L. Ong, and C. Brondos for technical and administrative assistance. We also thank Drs. Hao Shen, Allan J. Zajac, Linrong Lu, Lilin Ye, Jeffrey Frelinger, David Woodland, Marcia Blackman, Lubin Jiang, and Xiaolong Liu for their helpful comments, constructive suggestions and technical help. This work was also supported by the National Natural Science Foundation of China (grant No.:31570885 and 31270951), the Strategic Priority Research Program of Chinese Academy of Sciences (Grant No.: XDPB0303) and the Total Foundation. R.M.E. is an investigator of the Howard Hughes Medical Institute and March of Dimes Chair in Molecular and Developmental Biology at the Salk Institute. R.M.E. was funded by grants from NIH (DK057978, HL105278, DK090962, HL088093, ES010337, and CA014195) as well as the Helmsley Charitable Trust, Leducq Foundation, and Ipsen/Biomeasure. N.H. was supported by a T32 Institutional Research Training Grant (5T32CA009370). PBS57-loaded CD1d and unloaded mouse CD1d were provided by the NIH Tetramer Facility at Emory University.

\section{Author contributions}

J.W., N.H., R.M.E. and Q.L. designed the experiments, analyzed the data, wrote and edited the manuscript; J.W., N.H., N.Z., D.Q., Y.C., S.Z., C.Z., R.Z. and C.Y. performed the experiments; H.X. provided critical reagents and analyzed the data; and R.T.Y., A.R.A., C.L., M.D., Y.Z. and J.A. analyzed the data and edited the manuscript.

\section{Additional information}

Supplementary Information accompanies this paper at doi:10.1038/s41467-017-00931-8

Competing interests: The authors declare no competing financial interests.

Reprints and permission information is available online at http://npg.nature.com/ reprintsandpermissions/

Publisher's note: Springer Nature remains neutral with regard to jurisdictional claims in published maps and institutional affiliations.

Open Access This article is licensed under a Creative Commons Attribution 4.0 International License, which permits use, sharing, adaptation, distribution and reproduction in any medium or format, as long as you give appropriate credit to the original author(s) and the source, provide a link to the Creative Commons license, and indicate if changes were made. The images or other third party material in this article are included in the article's Creative Commons license, unless indicated otherwise in a credit line to the material. If material is not included in the article's Creative Commons license and your intended use is not permitted by statutory regulation or exceeds the permitted use, you will need to obtain permission directly from the copyright holder. To view a copy of this license, visit http://creativecommons.org/ licenses/by/4.0/

(c) The Author(s) 2017 